\title{
Pulsed laser crystallization of SiGe alloys on GaAs
}

\author{
F. Dondeo, ${ }^{1}$ P. V. Santos, ${ }^{2}$ M. Ramsteiner,${ }^{2}$ D. Comedi,${ }^{1}$ \\ M. A. A. Pudenzi, ${ }^{1}$, and I. Chambouleyron ${ }^{1}$ \\ ${ }^{1}$ Instituto de Física Gleb Wataghin, Universidade Estadual de Campinas, 13081-970 Campinas, SP, Brazil \\ 2 Paul-Drude-Institut für Festkörperelektronik, Hausvogteiplatz 5-7, 10117 Berlin, Germany
}

Received on 23 April, 2001

\begin{abstract}
We have investigated the crystallization of amorphous SiGe films deposited on crystalline GaAs (001) substrates using ns laser pulses. Analysis of the film structure using Raman spectroscopy indicates the formation of heteroepitaxial $\mathrm{Si}_{x} \mathrm{Ge}_{1-x} / \mathrm{GaAs}$ structures for $\mathrm{Si}$ compositions up to $x=$ 25\%. Higher compositions lead to polycrystalline films. This is attributed to the increased lattice mismatch between $\mathrm{Si}_{x} \mathrm{Ge}_{1-x}$ and GaAs as the Si fraction in the alloy increases.
\end{abstract}

\section{Introduction}

Pulsed laser crystallization (LC) [1] is a standard technique for the fabrication of large area polycrystalline films from the amorphous (a-) phase. This technique can also be used to produce high quality epitaxial Ge layers with abrupt interfaces on GaAs (001) substrates [2]. Since Ge and GaAs are well lattice-matched, an interesting question is how the epitaxial LC proceeds when $\mathrm{Si}$ is introduced in a controlled way into the $\mathrm{Ge}$ matrix, so as to form $\mathrm{Si}_{1-x} \mathrm{Ge}_{x}$ alloys with a lattice constant different from that of the substrate. In this contribution, we address this question by investigating the $\mathrm{LC}$ of a-Si $\mathrm{Ge}_{1-x}$ alloys deposited on GaAs (001) substrates. Raman scattering studies of the LC films give evidence for the epitaxial crystallization of $100-\mathrm{nm}-$ thick $\mathrm{Si}_{x} \mathrm{Ge}_{1-x}$ alloys with compositions $x$ up to $25 \%$. Higher compositions lead to polycrystalline films. This behavior is attributed to the increased lattice mismatch with the substrate, when the silicon concentration in the alloy increases. Since the epitaxial crystallization requires the melting of the amorphous film by the laser pulse, a second important issue regarding the crystallization of $\mathrm{Si}_{x} \mathrm{Ge}_{1-x}$ alloys on GaAs is the intermixing at the interface with the substrate. The intermixing was investigated using secondary ion mass spectrometry (SIMS).

\section{Experimental}

The $\mathrm{a}_{-} \mathrm{Si}_{x} \mathrm{Ge}_{1-x}$ samples were grown by ion-beamassisted sputtering on GaAs (001) substrates. Before film deposition, the substrates were heated to $350^{\circ} \mathrm{C}$ and bombarded by a $30 \mathrm{eV}$ hydrogen ion beam for $1 \mathrm{~min}$ in order to remove the natural surface oxide. Oxide removal was monitored in-situ using x-ray photoelectron spectroscopy. 100-nm-thick a-Si and a-Ge films were then deposited from $\mathrm{Si}$ and Ge targets (99.999\% pure) using $1000 \mathrm{eV} \mathrm{Kr}{ }^{+}$ions from a Kaufmann gun. $\mathrm{a}-\mathrm{Si}_{0.1} \mathrm{Ge}_{0.9}$ films of the same thickness were grown using a Ge target covered with a piece of Si. In order to obtain laser crystallized $\mathrm{Si}_{x} \mathrm{Ge}_{1-x}$ alloys with other compositions $x(x=0.25,0.50$, and 0.75$), \mathrm{a}-\mathrm{Si} / \mathrm{a}-\mathrm{Ge}$ multilayers with individual $\mathrm{Si}$ and Ge layer thicknesses below $25 \mathrm{~nm}$ were deposited. Each multilayer sample consists of 6 layers adding up to a total thickness of $100 \mathrm{~nm}$. As will be demonstrated below, the layers completely intermix during the laser crystallization process, leading to the formation of a homogeneous crystalline (c-) SiGe alloy with the composition determined by the relative thicknesses of the original a-Ge and a-Si layers.

The samples were crystallized with single pulses (pulse width of approx. $7 \mathrm{~ns}$ ) from a frequency-doubled Nd:Yag laser $(\lambda=532 \mathrm{~nm})$. A Gaussian-like laser beam profile with a diameter of approx. $4 \mathrm{~mm}$ was obtained by using a vacuum spatial filter. The laser pulse fluencies were estimated from the measured pulse energy by assuming a Gaussian profile for the distribution of light intensity on the irradiated area. The crystallization process was monitored in-situ by recording the transient reflectivity of a $\mathrm{cw}$ diode laser beam $(\lambda=675 \mathrm{~nm})$ during the LC process. The increase in the reflectivity indicates that the a-SiGe alloys melt during laser irradiation [3].

Raman spectroscopy was employed to study the structural properties of the samples. The measurements were performed at room temperature in the backscattering geometry using the $z(x, y) \bar{z}, z(y, y) \bar{z}$, $z\left(x^{\prime}, y^{\prime}\right) \bar{z}$, and $z\left(y^{\prime}, y^{\prime}\right) \bar{z}$ scattering configurations, where $x, y, z, \bar{z}, x^{\prime}$ and $y^{\prime}$ denote, respectively, the [100], [010], [001], [001] , [110], and [110] crystallographic di- 
rections of the (001) GaAs substrates. Note that deformation potential Raman scattering by longitudinal optical (LO) phonons is allowed by selection rules only for the configurations $z(x, y) \bar{z}$ and $z\left(y^{\prime}, y^{\prime}\right) \bar{z}[4]$. The red lines $\left(\lambda_{L}=676.4\right.$ or $\left.647.1 \mathrm{~nm}\right)$ of a $\mathrm{Kr}^{+}$-laser were used for excitation.

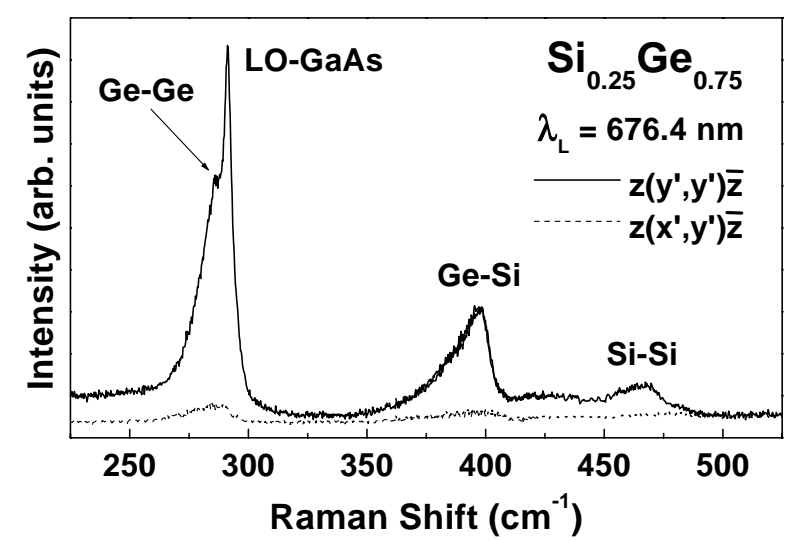

Figure 1. Raman spectra of a $\mathrm{LC} \quad \mathrm{c}_{-} \mathrm{Si}_{0.25} \mathrm{Ge}_{0.75}$ alloy recorded in the $z\left(y^{\prime}, y^{\prime}\right) \bar{z}$ (thick line, upper curve) and in the $z\left(x^{\prime}, y^{\prime}\right) \bar{z}$ (thin line, lower curve) scattering configurations. The laser fluency was approx. $500 \mathrm{~mJ} / \mathrm{cm}^{2}$.

\section{Results and Discussion}

The Raman spectra of as-grown a-SiGe multilayers show broad Raman lines centered around 270 and 470 $\mathrm{cm}^{-1}$, which are attributed to the $\mathrm{Ge}-\mathrm{Ge}$ and $\mathrm{Si}-\mathrm{Si}$ vibrations of the a-Ge and a-Si layers, respectively. After LC, these lines narrow and shift somewhat in energy. A Raman spectrum of a $\mathrm{LC} \mathrm{c}-\mathrm{Si}_{0.25} \mathrm{Ge}_{0.75}$ alloy with an average composition $x=25 \%$ recorded in the $z\left(y^{\prime}, y^{\prime}\right) \bar{z}$ configuration is shown by the solid line in Fig. 1 . The three main peaks found at 290,400 and $470 \mathrm{~cm}^{-1}$ correspond, respectively, to $\mathrm{Ge}-\mathrm{Ge}, \mathrm{Ge}-\mathrm{Si}$ and $\mathrm{Si}-\mathrm{Si}$ vibrations of a c- $\mathrm{Si}_{0.25} \mathrm{Ge}_{0.75}$ alloy [5]. The Ge-Si line, which only appears after LC, evidences the intermixing of the $\mathrm{Si}$ and Ge layers during laser irradiation. The sharp line at $292 \mathrm{~cm}^{-1}$ (close to the Ge-Ge vibration) corresponds to scattering by LO phonons in the GaAs substrate.

The spectrum recorded in the forbidden $z\left(x^{\prime}, y^{\prime}\right) \bar{z}$ configuration in Fig. 1 (thin line) shows a strongly reduced scattering intensity. This reduction is attributed to the epitaxial orientation of the $\mathrm{Si}_{0.25} \mathrm{Ge}_{0.75}$ film relative to the GaAs substrate. Similar results were also obtained for alloys with $x=10 \%$. Only polycrystalline regions were observed for $x>25 \%$, thus indicating that the large mismatch prevents the formation of epitaxial films.

The Raman spectrum and thus the structure of the LC layers depends sensitively on the laser pulse fluency. This result is illustrated in Fig. 2, which displays a sequence of Raman spectra recorded at different radial

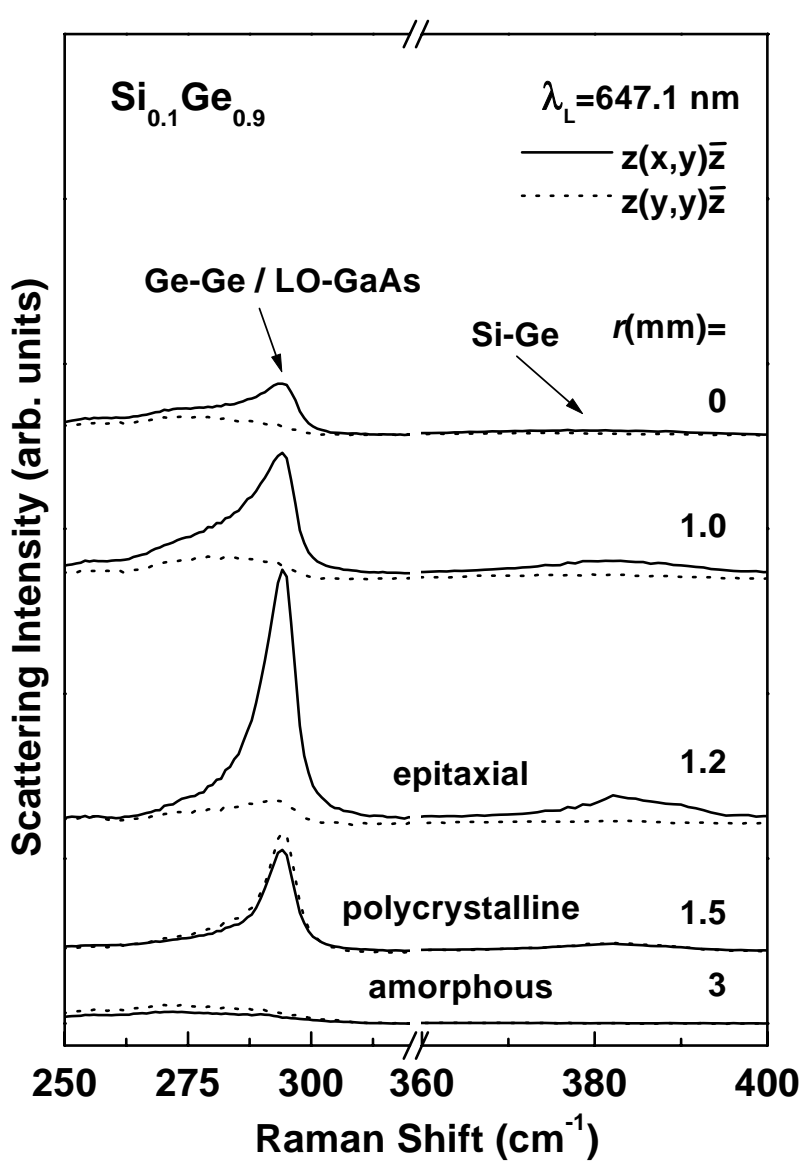

Figure 2. Raman spectra of a $\mathrm{LC} \mathrm{c-Si}{ }_{0.1} \mathrm{Ge}_{0.9}$ alloy recorded at different radial distances $r$ from the center of the LC spot. The fluencies for $x=0,1,1.2,1.5$, and $3 \mathrm{~mm}$ are estimated to be $2000,1250,1000,650$, and $0 \mathrm{~mJ} / \mathrm{cm}^{2}$.

distances $r$ from the center of the spot produced by LC on a multilayer with $x=0.10$. The laser pulse fluency decreases with $r$ following the Gaussian-like profile of the laser beam. For large distances $(r=3 \mathrm{~mm})$, the pulse energy is insufficient to induce the phase transition, so that the material remains amorphous. For $r=1.5 \mathrm{~mm}$, comparable intensities are measured in the $z(x, y) \bar{z}$ and $z(y, y) \bar{z}$ geometries, thus indicating the formation of a polycrystalline c-Si $\mathrm{Si}_{0.1} \mathrm{Ge}_{0.9}$ alloy. For $r<1.2 \mathrm{~mm}$, the ratio between the scattering intensities in the allowed and forbidden scattering geometries becomes very large. We attribute this large ratio to the formation of an epitaxial film with the same crystallographic orientation as the substrate.

The laser fluency of $1000 \mathrm{~mJ} / \mathrm{cm}^{2}$ estimated for $r=1.2$ is approximately the same as that required to obtain high quality LC-Ge films on GaAs. Although the material still crystallizes epitaxially for $r<1.2 \mathrm{~mm}$, the peak intensity of the Raman lines at approx. $290 \mathrm{~cm}^{-1}$ recorded in the allowed scattering configuration (thick line in Fig. 2) decreases with decreasing $r$. At the same time, the line broadens. This behavior is attributed to 


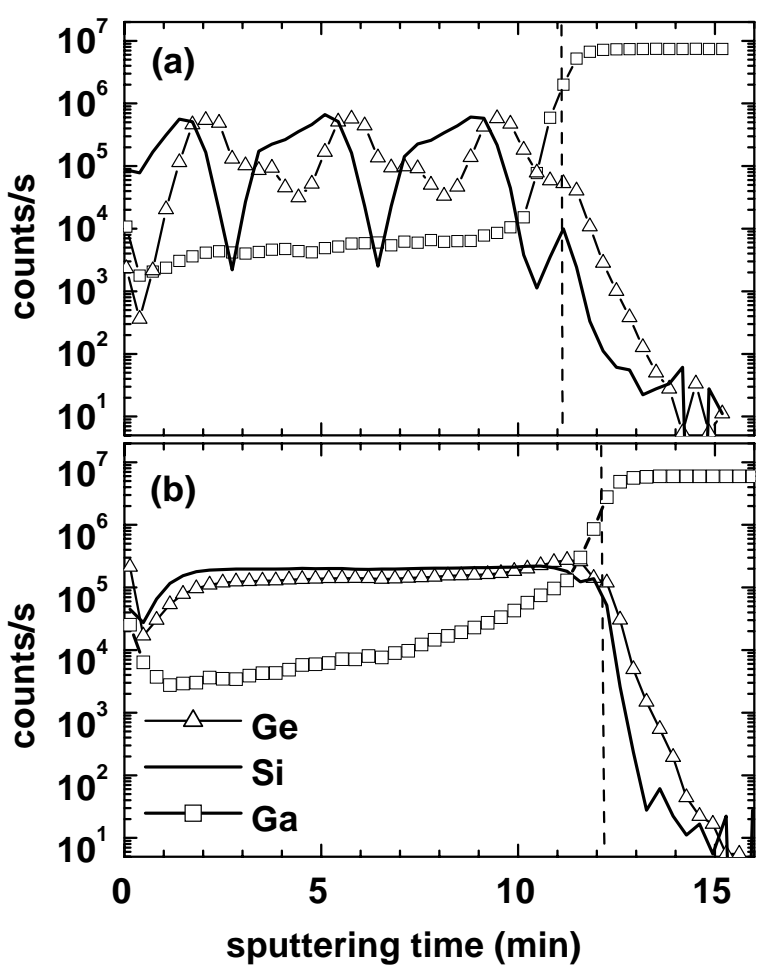

Figure 3. Concentrations of $\mathrm{Ge}, \mathrm{Si}$, and $\mathrm{Ga}$ as a function of SIMS sputtering time for an a-Si/a-Ge multilayer (a) before and (b) after LC. The composition of the $\mathrm{c}-\mathrm{Si}_{x} \mathrm{Ge}_{1-x}$ alloy is $x=0.25$. The original position of the interface with the substrate is indicated by the vertical dashed line.

the high temperatures induced in the $\mathrm{Si}_{1-x} \mathrm{Ge}_{x}$ layer under high laser fluencies, which leads to strong material intermixing at the $\mathrm{GaAs} / \mathrm{Si}_{1-x} \mathrm{Ge}_{x}$ interface and, eventually, to a partial melting of the underlying GaAs. When the $\mathrm{Si}_{1-x} \mathrm{Ge}_{x}$ alloy crystallizes, its absorption coefficient decreases, and it becomes almost transparent to the incident (and scattered) light. As a result, a substantial fraction of the Raman signal detected in LC films originates from the material close to the interface with the substrate. The broadened Raman line centered at $280 \mathrm{~cm}^{-1}$ is attributed to the scattering from coupled LO-phonon-plasmon modes in the intermixed p-type GaAs:Ge layer [6, 7].

The strong intermixing for high pulse fluencies becomes evident in the SIMS profiles displayed in Fig. 3, which were recorded on an a-Si/a-Ge multilayer before [Fig. 3(a)] and after LC [Fig. 3(b)]. The SIMS experiments were performed using oxygen as the primary ion beam. The dashed vertical lines indicate the original position of the $\mathrm{Si}_{0.25} \mathrm{Ge}_{0.75} /$ GaAs interface, located approx. $100 \mathrm{~nm}$ below the surface. The modulation of the $\mathrm{Ge}$ and $\mathrm{Si}$ concentrations in the as-grown multilayer is evident in Fig. 3(a). This modulation disappears as the individual $\mathrm{Si}$ and Ge layers completely intermix during $\mathrm{LC}$, giving rise to a homogeneous $\mathrm{c}-\mathrm{Si}_{0.25} \mathrm{Ge}_{0.75}$ film [Fig. 3(b)]. The profiles also show a strong interdiffusion of $\mathrm{Ga}$ into the $c-\mathrm{Si}_{0.25} \mathrm{Ge}_{0.75}$ film and a much less pronounced diffusion of $\mathrm{Ge}$ and $\mathrm{Si}$ into the substrate. Arsenic diffusion (not shown) was also observed in SIMS profiles measured with a cesium primary ion beam.

\section{Conclusions}

In conclusion, we have studied the structure of LC SiGe alloys grown on (001) GaAs substrates. Epitaxial films with a thickness of $100 \mathrm{~nm}$ were obtained for Si concentrations up to $25 \%$. Higher Si concentrations lead to polycrystalline films.

\section{Acknowledgments}

The investigation are partially supported by a DAAD (Germany) - CAPES (Brazil) international cooperation program.

\section{References}

[1] J. M. Poate and J.W. Mayer, Laser Annealing of Semiconductors (Academic Press, New York, 1982).

[2] J. E. Greene, K. C. Cadien, D. Lubben, G. A. Hawkins, G. Erikson, and J. R. Clarke, Appl. Phys. Lett. 39, 232 (1981).

[3] G. Aichmayr, D. Toet, M. Mulato, P. V. Santos, A. Spangenberg, S. Christiansen, M. Albrecht, and H. P. Strunk, J. Appl. Phys. 85, 1040 (1999).

[4] M. Cardona, Light Scattering in Solids II, ( Ed. M. Cardona and G. Güntherodt, Springer, Berlin, 1982), pp. 153 .

[5] M. A. Renucci, J. B. Renucci, and M. Cardona, Light Scattering in Solids, (Ed. M. Balkanski, Flammarion, Paris, 1971), pp. 326-329.

[6] A. Mlayah, R. Carles, and A. Leycuras, J. Appl. Phys. 71, 422 (1992).

[7] D. Olego and M. Cardona, Solid State Commun. 32, 375 (1979). 